\title{
MENGER MANIFOLDS HOMEOMORPHIC TO THEIR $n$-HOMOTOPY KERNELS
}

\author{
YUTAKA IWAMOTO
}

(Communicated by James West)

\begin{abstract}
We give a necessary and sufficient condition that an $(n+1)$-dimensional Menger manifold ( $\mu^{n+1}$-manifold) is homeomorphic to its $n$-homotopy kernel. Such a $\mu^{n+1}$-manifold is called a $\mu_{\infty}^{n+1}$-manifold. We also prove the following results:

(1) Each homeomorphism between two Z-sets in a $\mu_{\infty}^{n+1}$-manifold $M$ extends to an ambient homeomorphism of $M$ onto itself if it is $n$-homotopic to id in $M$.

(2) An $n$-homotopy equivalence between two $\mu_{\infty}^{n+1}$-manifolds is $n$-homotopic to a homeomorphism.

(3) Each map from a $\mu_{\infty}^{n+1}$-manifold into a $\mu^{n+1}$-manifold is $n$-homotopic to an open embedding.
\end{abstract}

\section{INTRODUCTION}

All spaces considered in this paper are assumed to be locally compact separable metrizable and maps are continuous. In [Be], Bestvina introduced Menger manifolds and established the characterization theorem of such manifolds. An $(n+1)$-dimensional Menger manifold is a topological manifold modeled on the $(n+1)$-dimensional universal Menger compactum $\mu^{n+1}$, which is also called a $\mu^{n+1}$-manifold. In [Ch3], Chigogidze introduced the notion of the $n$-homotopy kernel of a $\mu^{n+1}$-manifold and proved the following classification theorem for $\mu^{n+1}$-manifolds: Two $\mu^{n+1}$-manifolds have the same $n$-homotopy type if and only if their $n$-homotopy kernels are homeomorphic. There are close relations between Hilbert cube manifold ( $Q$-manifold) theory, and Menger manifold theory, and the $n$-homotopy kernel of a $\mu^{n+1}$-manifold plays the role of the product $X \times[0,1)$ of a $Q$-manifold $X$ with $[0,1)$. It is said that $X$ is $[0,1)$-stable if it is homeomorphic to $\cong) X \times[0,1)$.

Wong [Wo] showed that a $Q$-manifold $X$ is $[0,1)$-stable if and only if $X$ is properly contractible to $\infty$; that is, for any compactum $K$ in $X$ there is a proper map $j_{K}: X \rightarrow X \backslash K$ which is properly homotopic to $\mathrm{id}_{X}$. Replacing a proper homotopy with a proper $n$-homotopy, we have the notion of properly $n$-contractible to $\infty$. Moreover we say that $X$ is properly locally $(n$-)contractible

Received by the editors June 7, 1993.

1991 Mathematics Subject Classification. Primary 54F15, 54F35, 54F65.

Key words and phrases. Menger manifold, $n$-homotopy, $n$-homotopy kernel, properly $n$-contractible to $\infty$, properly locally $n$-contractible at $\infty, \mu_{\infty}^{n+1}$-manifold, $[0,1)$-stable $Q$-manifold. 
at $\infty$ if for any compactum $K \subset X$ there is a compactum $L \subset X$ with $K \subset L$ such that for each compactum $L^{\prime} \subset X$ with $L \subset L^{\prime}$ there exists a proper map $j_{L^{\prime}}: X \backslash L \rightarrow X \backslash L^{\prime}$ which is properly $\left(n\right.$-)homotopic to $\mathrm{id}_{X \backslash L}$ in $X \backslash K$. In this paper we define $\mu_{\infty}^{n+1}$-manifolds as $\mu^{n+1}$-manifolds which are properly $n$-contractible to $\infty$ and properly locally $n$-contractible at $\infty$ and show the following characterization theorem for $\mu_{\infty}^{n+1}$-manifolds.

Theorem I. Let $M$ be a $\mu^{n+1}$-manifold. Then $M$ is a $\mu_{\infty}^{n+1}$-manifold if and only if $M$ is homeomorphic to its n-homotopy kernel $\operatorname{Ker}(M)$.

We will show that two $n$-homotopic proper maps into a $\mu_{\infty}^{n+1}$-manifold are properly $n$-homotopic (see Lemma 3.1). Thus we can remove the requirement of an $n$-homotopy between $\mu_{\infty}^{n+1}$-manifold to be proper, whence we obtain the following Z-set unknotting theorem for $\mu_{\infty}^{n+1}$-manifolds.

Theorem II. Each homeomorphism between two Z-sets in a $\mu_{\infty}^{n+1}$-manifold $M$ extends to an ambient homeomorphism of $M$ onto itself if it is n-homotopic to id in $M$.

From Theorem 2.2 in [Ch3], it follows that two $\mu_{\infty}^{n+1}$-manifolds of the same $n$-homotopy type are homeomorphic. Similarly to [C1, Theorem 5], we can clarify the relation between $n$-homotopy equivalences and homeomorphisms, that is:

Theorem III. An n-homotopy equivalence between two $\mu_{\infty}^{n+1}$-manifolds is $n$ homotopic to a homeomorphism.

Moreover, similarly to $[0,1)$-stable $Q$-manifolds [C1, Lemma 3.6], we can strengthen the open embedding theorem [Ch 2, Ch 3].

Theorem IV. Each map from a $\mu_{\infty}^{n+1}$-manifold into a $\mu^{n+1}$-manifold is $n$-homotopic to an open embedding.

\section{Preliminaries}

We say two (proper) maps $f, g: X \rightarrow Y$ are (properly) n-homotopic (notation: $f \simeq^{n} g, f \simeq_{p}^{n} g$, respectively) if, for any (proper) map $\alpha: Z \rightarrow X$ from a space $Z$ with $\operatorname{dim} Z \leq n$ into $X$, the compositions $f \alpha$ and $g \alpha$ are (properly) homotopic in the usual sense. The notion of $n$-homotopy equivalence is defined in the obvious way.

Proposition 1.1 [Hu]. Let $f: X \rightarrow Y$ be a map, where $\operatorname{dim} X \leq n$ and $Y$ is $L C^{n}$. Then for any open cover $\mathscr{U}$ of $Y$, there are maps $\varphi: X \rightarrow P$ and $\psi: P \rightarrow Y$ such that $f$ and $\psi \varphi$ are $\mathscr{U}$-homotopic, where $P$ is a locally finite polyhedron with $\operatorname{dim} P \leq n$. In particular, we can choose $\psi$ as a proper map.

Let us recall that a map $f: X \rightarrow Y$ is said to be $n$-invertible if for any space $Z$ with $\operatorname{dim} Z \leq n$ and any map $\alpha: Z \rightarrow Y$ there exists a map $\beta: Z \rightarrow X$ such that $f \beta=\alpha$.

Proposition 1.2 [Ch2]. Every $\mu^{n+1}$-manifold admits a proper $(n+1)$-invertible $U V^{n}$-surjection onto a Q-manifold.

Proposition 1.3 [Ch3]. Two $\mu^{n+1}$-manifolds admitting proper $U V^{n}$-surjections onto the same $L C^{n}$-space are homeomorphic. 
The following theorem is due to Bestvina [Be], where it is stated in terms of $\mu$-homotopy. However, as is known [Ch1], the notion of $\mu$-homotopy coincides with one of $n$-homotopy for maps between locally compact $L C^{n}$-spaces of dimension at most $n+1$.

Theorem 1.1 (Z-set unknotting theorem). Let $M$ be a $\mu^{n+1}$-manifold and $f$ : $A \rightarrow B$ be a homeomorphism between Z-sets in $M$. If $f \simeq_{p}^{n} \operatorname{id}_{A}$ in $M$, then $f$ extends to a homeomorphism $h: M \rightarrow M$.

An $n$-homotopy kernel of a $\mu^{n+1}$-manifold $M$ is defined to be the complement $M \backslash f(M)$ of the image of an arbitrary Z-embedding $f: M \rightarrow M$ with $f \simeq_{p}^{n} \mathrm{id}_{M}$. Using the Z-set unknotting theorem, two $n$-homotopy kernels are homeomorphic by an ambient homeomorphism of $M$ onto itself. By $\operatorname{Ker}(M)$, we denote a representative of $n$-homotopy kernels of $M$. The following proposition is actually proved in [Ch3].

Proposition 1.4. For each $\mu^{n+1}$-manifold $M$ there exists a proper $(n+1)$ invertible $U V^{n}$-surjection $f: M \rightarrow M \times[0,1]$ such that $f^{-1}(M \times[0,1))=$ $\operatorname{Ker}(M)$.

Theorem 1.2 [Dr]. There exists an $(n+1)$-invertible $U V^{n}$-surjection $f_{n}: \mu^{n+1} \rightarrow$ $Q$ satisfying the following condition:

(*) $f_{n}^{-1}(X)$ is a $\mu^{n+1}$-manifold for any locally compact $L C^{n}$-space $X \subset Q$.

Theorem 1.3 [Ch4]. For each locally finite polyhedron $K$, there exists a proper $(n+1)$-invertible $U V^{n}$-surjection $f_{K}: M_{K} \rightarrow K$ from a $\mu^{n+1}$-manifold $M_{K}$ onto $K$ satisfying the following conditions:

(a) $f_{K}^{-1}(L)$ is a $\mu^{n+1}$-manifold for any closed subpolyhedron $L$ of $K$;

(b) $f_{K}^{-1}(Z)$ is a $Z$-set in $f_{K}^{-1}(L)$ for any $Z$-set $Z$ in a closed subpolyhedron $L$ of $K$.

Let $f: X \rightarrow Y$ be a proper map. We say that $f$ induces an epimorphism of $j^{\text {th }}$ homotopy groups of ends if for every compactum $C \subset Y$ there exists a compactum $K \subset Y$ such that for each point $x \in X \backslash f^{-1}(K)$ and every map $\alpha:\left(S^{j}, *\right) \rightarrow(Y \backslash K, f(x))$ there exist a map $\tilde{\alpha}:\left(S^{j}, *\right) \rightarrow\left(X \backslash f^{-1}(C), x\right)$ and a homotopy $f \tilde{\alpha} \simeq \alpha$ rel. $*$ in $Y \backslash C$. Also we say that $f$ induces $a$ monomorphism of $j^{\text {th }}$ homotopy groups of ends if for every compactum $C \subset Y$ there exists a compactum $K \subset Y$ such that for every map $\tilde{\alpha}: S^{j} \rightarrow X \backslash f^{-1}(K)$ with $f \tilde{\alpha} \simeq *$ in $Y \backslash K$ it follows that $\tilde{\alpha} \simeq *$ in $X \backslash f^{-1}(C)$. It is said that $f$ induces an isomorphism of $j^{\text {th }}$ homotopy groups of ends if $f$ induces both the epimorphism and monomorphism of $j^{\text {th }}$ homotopy groups of ends.

Theorem 1.4 [Be]. Let $f: M \rightarrow N$ be a proper map between $\mu^{n+1}$-manifolds. If $f$ induces an isomorphism of homotopy groups of $\operatorname{dim} \leq n$ and an isomorphism of homotopy groups of ends of $\operatorname{dim} \leq n$, then $f$ is properly $n$-homotopic to a homeomorphism.

\section{Characterization of $\mu_{\infty}^{n+1}$-MANifolds}

A space $X$ is said to be properly ( $n$-) contractible to $\infty$ if for any compactum $K$ in $X$ there exists a proper map $j_{K}: X \rightarrow X \backslash K$ which is properly 
( $n$-)homotopic to $\mathrm{id}_{X}$. If for any compactum $K \subset X$ there exists a compactum $L \subset X$ with $K \subset L$ such that for each compactum $L^{\prime} \subset X$ with $L \subset L^{\prime}$ there exists a proper map $j_{L^{\prime}}: X \backslash L \rightarrow X \backslash L^{\prime}$ which is properly ( $n$-)homotopic to $\operatorname{id}_{X \backslash L}$ in $X \backslash K$, then a space $X$ is said to be properly locally ( $n$-)contractible at $\infty$. It is easy to see that for any space $X, X \times[0,1)$ is properly contractible to $\infty$ and properly locally contractible at $\infty$.

Lemma 2.1. Let $X$ be properly $n$-contractible to $\infty$ and properly locally $n$ contractible at $\infty$. Then for each compact cover $\left\{X_{i}\right\}_{i \in \omega}$ of $X$ with $X_{i} \subset$ int $X_{i+1}$, there exist a subcover $\left\{X_{i_{k}} \mid k \in \omega, 0=i_{0}<i_{1}<i_{2}<\cdots\right\}$ and a collection of proper maps $\left\{f_{k}: X \rightarrow X \backslash X_{i_{k}}\right\}_{k \in \omega}$ such that $f_{0}=\operatorname{id}_{X}$ and $f_{k-1} \simeq_{p}^{n} f_{k}$ in $X \backslash X_{i_{k-2}}$ for $k \geq 1$, where $X_{i_{-1}}=\varnothing$.

Proof. For technical reasons we assume that $X_{0}=\varnothing$. Let $L_{-2}=L_{-1}=L_{0}=$ $\varnothing$. We shall inductively choose integers $0=i_{-2}=i_{-1}=i_{0}<i_{1}<i_{2}<\cdots$ and construct compacta $L_{k-1} \subset X_{i_{k}} \subset L_{k}$ and proper maps $j_{k}: X \backslash L_{k-2} \rightarrow X \backslash X_{i_{k}}$, $k \in \omega$, satisfying the following conditions:

(1) $j_{0}=\mathrm{id}_{X}$.

(2) For each compactum $M \supset L_{k}$ there is a proper map $j_{M}: X \backslash L_{k} \rightarrow$ $X \backslash M$ such that $j_{M} \simeq_{p}^{n} \operatorname{id}_{X \backslash L_{k}}$ in $X \backslash X_{i_{k-2}}$.

(3) $j_{k} \simeq \simeq_{p}^{n} \operatorname{id}_{X \backslash L_{k-2}}$ in $X \backslash X_{i_{k-2}}$.

Let $i_{1}=1$. Since $X$ is properly $n$-contractible to $\infty$ and properly locally $n$ contractible at $\infty$, there exist a proper map $j_{1}: X \rightarrow X \backslash X_{i_{1}}$ with $j_{1} \simeq_{p}^{n}$ id and a compactum $L_{1} \supset X_{1}$ satisfying (2). Since $X=\cup_{i \in \omega} X_{i}$ and $X_{i} \subset$ int $X_{i+1}$, there exists $i_{2}>i_{1}$ such that $X_{i_{2}} \supset L_{1}$. As in the above arguments there exist a proper map $j_{2}: X \rightarrow X \backslash X_{i_{2}}$ with $j_{2} \simeq_{p}^{n} \operatorname{id}_{X}$ and a compactum $L_{2} \supset X_{i_{2}}$ satisfying (2).

Assume that, for $k \geq 2, i_{0}<i_{1}<\cdots<i_{k}, L_{k}$, and $j_{k}: X \backslash L_{k-2} \rightarrow$ $X \backslash X_{i_{k}}$ have been constructed. Choose $i_{k+1}>i_{k}$ so that $X_{i_{k+1}} \supset L_{k}$. Since $X_{i_{k+1}} \supset L_{k-1}$, by the property (2) of $L_{k-1}$, there exists a proper map $j_{X_{i_{k+1}}}$ : $X \backslash L_{k-1} \rightarrow X \backslash X_{i_{k+1}}$ such that $j_{X_{i_{k+1}}} \simeq_{p}^{n} \operatorname{id}_{X \backslash L_{k-1}}$ in $X \backslash X_{i_{k-1}}$. Then put $j_{k+1}=j_{X_{i_{k+1}}}$. Since $X$ is properly locally $n$-contractible at $\infty$, there exists a compactum $L_{k+1} \supset X_{i_{k+1}}$ satisfying (2).

Now define $f_{k}=j_{k} \cdots j_{0}: X \rightarrow X \backslash X_{i_{k}}$ for $k \in \omega$ and observe that the collections of compacta $\left\{X_{i_{k}}\right\}_{k \in \omega}$ and maps $\left\{f_{k}\right\}_{k \in \omega}$ are as desired.

As is stated in the introduction, a $\mu_{\infty}^{n+1}$-manifold is a $\mu^{n+1}$-manifold which is properly $n$-contractible to $\infty$ and properly locally $n$-contractible at $\infty$. Theorem $I$ is contained in the following.

Theorem 2.1 (Characterization). For a $\mu^{n+1}$-manifold $M$ the following conditions are equivalent:

(1) $M$ is a $\mu_{\infty}^{n+1}$-manifold.

(2) $M \cong \operatorname{Ker}(M)$.

(3) There is a proper $(n+1)$-invertible $U V^{n}$-surjection $f: M \rightarrow X$ onto some $[0,1)$-stable $Q$-manifold $X$.

(4) There is a proper $(n+1)$-invertible $U V^{n}$-surjection $g: M \rightarrow Y$ onto a space $Y$ which is properly $n$-contractible to $\infty$ and properly locally $n$-contractible at $\infty$. 
Proof. We shall prove that $(1) \Rightarrow(2)$. First we shall choose a compact cover $\left\{M_{i}\right\}_{i \in \omega}$ of $M$ with $M_{i} \subset$ int $M_{i+1}, \quad i \in \omega$, such that the topological frontier Fr $M_{i}$ is a Z-set in $M \backslash$ int $M_{i}$. To this end, fix a proper $U V^{n}$-surjection $g$ : $M \rightarrow X$ onto a $Q$-manifold $X$. Then choose a compact cover $\left\{X_{i}\right\}_{i \in \omega}$ of $X$ consisting of $Q$-manifold with $X_{i} \subset$ int $X_{i+1}$ such that $\operatorname{Fr} X_{i}$ is a Z-set in both $X_{i}$ and $X \backslash$ int $X_{i}, \quad i \in \omega$ (see [C2, CS]). For each $i \in \omega$, by the relative triangulation theorem for $Q$-manifolds [C3], we may assume that $X=P \times Q$, $X_{i}=P_{1}^{i} \times Q$, and $X \backslash$ int $X_{i}=P_{2}^{i} \times Q$ for a locally finite polyhedron $P$ and closed subpolyhedra $P_{1}^{i}, \quad P_{2}^{i} \subset P$. Note that $P_{1}^{i} \cap P_{2}^{i}$ is a Z-set in $P_{2}^{i}$. Let $f_{P}: M_{P} \rightarrow P$ be a proper $U V^{n}$-surjection from a $\mu^{n+1}$-manifold $M_{P}$ onto $P$ satisfying condition (b) in Theorem 1.3. Since the composition $\pi_{P} g: M \rightarrow P$ is proper $U V^{n}$ (where $\pi_{P}: P \times Q \rightarrow P$ is the canonical projection), there is a homeomorphism $k: M_{P} \rightarrow M$ by Proposition 1.3. Then by property (b) of $f_{P}, f_{P}^{-1}\left(P_{1}^{i} \cap P_{2}^{i}\right)$ is a Z-set in $f_{P}^{-1}\left(P_{2}^{i}\right)$ and so is the topological frontier of $f_{P}^{-1}\left(P_{1}^{i}\right)$. Now let $M_{i}=k f_{P}^{-1}\left(P_{1}^{i}\right), \quad i \in \omega$. Then the compact cover $\left\{M_{i}\right\}_{i \in \omega}$ of $M$ is the required one.

By Lemma 2.1, there is a collection of maps $\left\{f_{i}: M \rightarrow M \backslash M_{i}\right\}_{i \in \omega}$ such that $f_{0}=\operatorname{id}_{M}, f_{i} \simeq_{p}^{n} f_{i+1}$ in $M \backslash M_{i-1}$ for $i \in \omega$. Using the Z-embedding approximation theorem for $\mu^{n+1}$-manifolds [Be, 2.3.8], we can choose $f_{i}$ as a Z-embedding for each $i \in \omega$. Put $K_{i}=M \backslash f_{i}(M)$ for $i \geq 1$. Then since $f_{i} \simeq_{p}^{n} \mathrm{id}_{M}$, by the definition of $n$-homotopy kernels, we have $K_{i} \cong \operatorname{Ker}(M)$. By Theorem 1.1, since $f_{i} \simeq_{p}^{n} f_{i+1}$ is in $M \backslash M_{i-1}$ and $\operatorname{Fr} M_{i-1}$ is a Z-set in $M \backslash \operatorname{int} M_{i-1}$, there exists a homeomorphism $h_{i}: M \rightarrow M$ such that $h_{i} f_{i}=f_{i+1}$ and $\left.h_{i}\right|_{M_{i-1}}=\mathrm{id}$. Note that $h_{i}\left(K_{i}\right)=K_{i+1}$. Now we define $h: K_{1} \rightarrow M$ by $h=\lim _{i \rightarrow \infty} h_{i} \cdots h_{1}$. Then $\left.h\right|_{h^{-1}\left(\text { int } M_{i}\right)}=\left.h_{i+2} \cdots h_{1}\right|_{h^{-1}\left(\text { int } M_{i}\right)}$. In fact, suppose that $h(x) \neq h_{i+2} \cdots h_{1}(x)$ for some $x \in h^{-1}$ (int $\left.M_{i}\right)$. Then there is an open subset $U$ of int $M_{i}$ such that $h(x) \in U \subset \bar{U}$ and $h_{i+2} \cdots h_{1}(x) \notin \bar{U}$. Since $\left.h_{j}\right|_{\text {int } M_{i}}=$ id for $j \geq i+2, h_{j} \cdots h_{1}(x)=h_{i+2} \cdots h_{1}(x) \notin \bar{U}$ for all $j \geq i+2$. This contradicts the definition of $h$.

One can easily see that $h$ is injective. Moreover, since $M=\cup_{i \in \omega} M_{i}$ and $h_{i} \cdots h_{1}\left(K_{1}\right)=K_{i} \supset M_{i}$, it follows that $h$ is surjective. To finish the proof, it only remains to note that $h$ is open. Thus $h$ is a homeomorphism.

To prove $(2) \Rightarrow(3)$, assume $M \cong \operatorname{Ker}(M)$. Then, by Proposition 1.4, there is a proper $(n+1)$-invertible $U V^{n}$-surjection $g: M \rightarrow M \times[0,1)$. Let $h: M \rightarrow Y$ be a proper $U V^{n}$-surjection onto a $Q$-manifold $Y$ (Proposition 1.2). Since $Y \times[0,1)$ is a $[0,1)$-stable $Q$-manifold, the composition $\left(h \times \mathrm{id}_{[0,1)}\right) g: M \rightarrow Y \times[0,1)$ is the required one.

(3) $\Rightarrow(4)$ is trivial.

Finally we shall show that $(4) \Rightarrow(1)$. Let $h: M \rightarrow X$ be a proper $(n+1)$-invertible $U V^{n}$-surjection onto a space $X$ properly $n$-contractible to $\infty$ and properly locally $n$-contractible at $\infty$. Let $K$ be a compactum in $M$. Then there exists a compactum $L^{\prime}$ in $X$ with $h(K) \subset L^{\prime}$ such that for each compactum $F^{\prime}$ with $L^{\prime} \subset F^{\prime}$ there exist proper maps $i_{h(K)}^{\prime}: X \rightarrow X \backslash h(K)$ and $j_{F^{\prime}}^{\prime}: X \backslash L^{\prime} \rightarrow X \backslash F^{\prime}$ such that $i_{h(K)}^{\prime} \simeq_{p}^{n} \operatorname{id}_{X}$ in $X$ and $j_{F^{\prime}}^{\prime} \simeq_{p}^{n} \operatorname{id}_{X \backslash L^{\prime}}$ in $X \backslash h(K)$. Let $L=h^{-1}\left(L^{\prime}\right)$ and $F$ be a compactum containing $L$. Since $h$ is proper $(n+1)$-invertible, there exist proper maps $i_{K}: M \rightarrow M \backslash K$ and $j_{F}: M \backslash L \rightarrow M \backslash F$ such that $h i_{K}=i_{h(K)}^{\prime} h$ and $h j_{F}=j_{h(F)}^{\prime} h$. 
Consider a proper map $\alpha: Z \rightarrow M \backslash L\left(\subset M \backslash h^{-1} h(K)\right)$, where $\operatorname{dim} Z \leq n$. We shall now show that $j_{F} \alpha$ is properly homotopic to $\alpha$ in $M \backslash K$. From Proposition 1.1, we may assume without loss of generality that $Z$ is a locally finite polyhedron. Let $H:\left(X \backslash L^{\prime}\right) \times[0,1] \rightarrow X \backslash h(K)$ be a proper homotopy from $\mathrm{id}_{X \backslash L^{\prime}}$ to $j_{h(F)}^{\prime}$. Then $H(h \alpha \times \mathrm{id}): Z \times[0,1] \rightarrow X \backslash h(K)$ is a proper homotopy from $h \alpha$ to $j_{h(F)}^{\prime} h \alpha=h j_{F} \alpha$. Since $\left.h\right|_{M \backslash h^{-1} h(K)}: M \backslash h^{-1} h(K) \rightarrow$ $X \backslash h(K)$ is proper $U V^{n}$, by [La, $\S 3$, Lemma A], there exists a proper homotopy $F: Z \times[0,1] \rightarrow M \backslash h^{-1} h(K)$ from $\alpha$ to $j_{F} \alpha$. Thus $j_{F} \simeq_{p}^{n} \operatorname{id}_{M \backslash L}$ in $M \backslash K$. Similarly, we can conclude $i_{F} \simeq_{p}^{n} \operatorname{id}_{M}$.

\section{Proofs of Theorems II, III, AND IV}

Lemma 3.1. Let $f: X \rightarrow Y$ be a map from a locally compact space $X$ into a $L C^{n}$-space $Y$ admitting a proper $(n+1)$-invertible $U V^{n}$-surjection onto a space $Y \times[0,1)$. Then $f$ is $n$-homotopic to a proper map whenever $\operatorname{dim} X \leq n+1$. Moreover, if $f$ is a proper map n-homotopic to a proper map $g: X \rightarrow Y$, then $f \simeq_{p}^{n} g$.

Proof. Fix a proper map $p: X \rightarrow[0,1)$, and let $h: Y \rightarrow Y \times[0,1)$ be a proper $(n+1)$-invertible $U V^{n}$-surjection. Let $q: X \rightarrow Y \times[0,1)$ be the map defined by $q(x)=\left(h_{1} f(x), p(x)\right)$, where $h(x)=\left(h_{1}(x), h_{2}(x)\right), x \in X$. Then $q$ is proper and homotopic to $h f$. By the $(n+1)$-invertibility of $h$, there is a map $f^{\prime}: X \rightarrow Y$ such that $h f^{\prime}=q$. Note that $f^{\prime}$ is proper and $h f^{\prime} \simeq h f$. Thus by the lifting property of $h\left[\mathrm{La}, \S 3\right.$, Lemma A], we conclude that $f \simeq^{n} f^{\prime}$.

Next suppose that $f$ is a proper map $n$-homotopic to a proper map $g: X \rightarrow$ $Y$. Let $\alpha: Z \rightarrow X$ be a proper map, where $\operatorname{dim} Z \leq n$. We shall show that $f \alpha \simeq_{p} g \alpha$. By Proposition 1.1, we may assume without loss of generality that $Z$ is a locally finite polyhedron. Let $\left\{Y_{i}\right\}_{i \in \omega}$ be a compact cover of $Y$ with $Y_{0}=\varnothing$ and $Y_{i} \subset$ int $Y_{i+1}, i \in \omega$. Then for each $i \geq 1$, let $Z_{i}$ be a compact subpolyhedron of $Z$ such that $(h f \alpha)^{-1}\left(W_{i}\right) \cup(h g \alpha)^{-1}\left(W_{i}\right) \subset Z_{i} \subset$ int $Z_{i+1}$, where $Z_{0}=\varnothing$ and $W_{i}=Y_{i} \times\left[0,1-2^{-i}\right]$. Since $f \simeq^{n} g$, we can fix a homotopy $G_{0}: Z \times[0,1] \rightarrow Y$ from $f \alpha$ to $g \alpha$. For $k \geq 1$, we shall inductively construct a homotopy $G_{k}:\left(Z \backslash\right.$ int $\left.Z_{2 k-2}\right) \times[0,1] \rightarrow Y \backslash h^{-1}\left(W_{2 k-5}\right)$ from the restriction $f \alpha \mid$ of $f \alpha$ to the one $g \alpha \mid$ of $g \alpha$ satisfying the following conditions:

$(1)_{k} G_{k}\left(\left(Z \backslash\right.\right.$ int $\left.\left.Z_{2 k}\right) \times[0,1]\right) \subset Y \backslash h^{-1}\left(W_{2 k-2}\right)$;

$(2)_{k} \quad G_{k}=G_{k-1}$ on $\operatorname{Fr} Z_{2 k-2} \times[0,1]$.

Let $F_{i}:[0,1) \rightarrow\left[1-2^{-i}, 1\right)$ be the map defined by $F_{i}(t)=1+(t-1) 2^{-i}$ for each $i \geq 1$. Suppose that a homotopy $G_{k}:\left(Z \backslash\right.$ int $\left.Z_{2 k-2}\right) \times[0,1] \rightarrow$ $Y \backslash h^{-1}\left(W_{2 k-5}\right)$ has been constructed for $k \in \omega$. Then let $A_{k+1}=\left(Z \backslash \operatorname{int} Z_{2 k}\right) \times$ $\{0,1\} \cup \operatorname{Fr} Z_{2 k} \times[0,1]$ and $B_{k+1}=\left(h G_{k}\right)^{-1}\left(W_{2 k+1}\right) \cap\left(Z \backslash\right.$ int $\left.Z_{2 k+2}\right) \times[0,1]$. Since $A_{k+1}$ and $B_{k+1}$ are disjoint closed, we can choose $\beta:\left(Z \backslash\right.$ int $\left.Z_{2 k}\right) \times$ $[0,1] \rightarrow[0,1]$ such that $\beta\left(A_{k+1}\right)=0$ and $\beta\left(B_{k+1}\right)=1$. Define $G_{k+1}^{\prime}$ : $\left(Z \backslash\right.$ int $\left.Z_{2 k}\right) \times[0,1] \rightarrow Y \times[0,1) \backslash W_{2 k-2}$ by

$$
G_{k+1}^{\prime}(w)=\left(s_{k}(w),(1-\beta(w)) t_{k}(w)+\beta(w) F_{2 k+2} t_{k}(w)\right),
$$

where $h G_{k}(w)=\left(s_{k}(w), t_{k}(w)\right), w \in\left(Z \backslash\right.$ int $\left.Z_{2 k}\right) \times[0,1]$. By the lifting property [La], there is a homotopy $G_{k+1}:\left(Z \backslash\right.$ int $\left.Z_{k}\right) \times[0,1] \rightarrow Y \backslash W_{2 k-3}$ from $f \alpha \mid$ to $g \alpha \mid$ with $h G_{k+1}=G_{k+1}^{\prime}$ and $G_{k+1}=G_{k}$ on $A_{k+1}$ (i.e., satisfying $\left.(2)_{k+1}\right)$ such that $G_{k+1}$ satisfies $(1)_{k+1}$. 
We define $H: Z \times[0,1] \rightarrow Y$ by $H=G_{k}$ on each $\left(Z_{2 k} \backslash\right.$ int $\left.Z_{2 k-2}\right) \times[0,1]$. Then $H$ is a well-defined homotopy from $f \alpha$ to $g \alpha$. Note that since $h$ is proper, $\left\{h^{-1}\left(W_{i}\right)\right\}_{i \in \omega}$ is a compact cover of $Y$ with $h^{-1}\left(W_{i}\right) \subset \operatorname{int} h^{-1}\left(W_{i+1}\right)$. Thus it follows from our construction that $H$ is proper. The proof is finished.

Proof of Theorem II. The theorem directly follows from Theorem 1.1 and Lemma 3.1.

Lemma 3.2. If $f: M \rightarrow N$ is a proper $n$-homotopy equivalence between $\mu_{\infty}^{n+1}$ manifolds, then $f$ induces an isomorphism of homotopy groups of ends of $\operatorname{dim} \leq$ $n$.

Proof. By Theorem 2.1, we can fix proper $(n+1)$-invertible $U V^{n}$-surjections $g: M \rightarrow X \times[0,1)$ and $h: N \rightarrow Y \times[0,1)$, where $X$ and $Y$ are some $Q$-manifolds. Let $C$ be a compactum in $N$. Then there is a compactum $C^{\prime \prime} \subset Y$ such that $C^{\prime \prime} \times\left[0, t^{\prime}\right] \supset h(C)$ for some $t^{\prime} \in(0,1)$. Since $h$ is proper, $C^{\prime}=h^{-1}\left(C^{\prime \prime} \times\left[0, t^{\prime}\right]\right)$ is a compactum with $C^{\prime} \supset C$. Note that, since $f$ is proper, $g\left(f^{-1}\left(C^{\prime}\right)\right)$ is a compactum in $X \times[0,1)$. Thus there exists $t_{1} \in(0,1)$ such that $L=\pi_{X}\left(g\left(f^{-1}\left(C^{\prime}\right)\right)\right) \times\left[0, t_{1}\right] \supset g\left(f^{-1}\left(C^{\prime}\right)\right)$, where $\pi_{X}: X \times[0,1) \rightarrow X$ is the canonical projection. Similarly, since $g$ is proper, there exists $t_{2} \in(0,1)$ such that $K^{\prime}=\pi_{Y}\left(h f\left(g^{-1}(L)\right)\right) \times\left[0, t_{2}\right] \supset h f\left(g^{-1}(L)\right)$, where $\pi_{Y}: Y \times[0,1) \rightarrow Y$ is the canonical projection. Put $K=h^{-1}\left(K^{\prime}\right)$, and let $x_{0} \in M \backslash f^{-1}(K), j \leq n$, and $\alpha:\left(S^{j}, *\right) \rightarrow\left(N \backslash K, f\left(x_{0}\right)\right)$. Since $f$ is an $n$-homotopy equivalence, there exists $\alpha_{1}:\left(S^{j}, *\right) \rightarrow\left(M, x_{0}\right)$ such that $f \alpha_{1} \simeq \alpha$ rel.*. Since $\alpha_{1}^{-1}\left(x_{0}\right)$ and $\alpha_{1}^{-1}\left(g^{-1}(L)\right)$ are disjoint closed sets in $S^{j}$, we can choose a map $\beta: S^{j} \rightarrow[0,1]$ such that $\beta\left(\alpha_{1}^{-1}\left(x_{0}\right)\right)=0$ and $\beta\left(\alpha_{1}^{-1}\left(g^{-1}(L)\right)\right)=1$. Say $g \alpha_{1}(x)=\left(\pi_{X} g \alpha_{1}(x), t(x)\right) \in X \times[0,1), x \in S^{j}$. Define $\alpha_{2}:\left(S^{j}, *\right) \rightarrow\left(X \times[0,1), g\left(x_{0}\right)\right)$ by

$$
\alpha_{2}(x)=\left(\pi_{X} g \alpha_{1}(x),\left(1-t_{1}\right) \cdot t(x)+t_{1}\right) \beta(x)+(1-\beta(x) \cdot t(x)), \quad x \in S^{j} .
$$

Clearly $\alpha_{2} \simeq g \alpha_{1}$ rel. $*$ and $\alpha_{2}\left(S^{j}\right) \cap L=\varnothing$. Using the lifting property [La] of the proper $U V^{n}$-surjection $g$, there exists $\tilde{\alpha}:\left(S^{j}, *\right) \rightarrow\left(M, x_{0}\right)$ such that $\operatorname{img} \tilde{\alpha} \cap L=\varnothing$ and $\tilde{\alpha} \simeq \alpha_{1}$ rel. *. Hence we have $f \tilde{\alpha} \simeq \alpha$ rel. $*$ and $f \tilde{\alpha}\left(S^{j}\right) \cap C^{\prime}=\varnothing$. By the same technique we performed above, we can choose a homotopy so that $f \tilde{\alpha} \simeq \alpha$ rel. $*$ in $N \backslash C$.

Next let $\gamma: S^{j} \rightarrow M \backslash f^{-1}(K)$ be a map such that $f \gamma \simeq *$ in $N \backslash K$. Since $f$ is an $n$-homotopy equivalence, $g \gamma \simeq *$ in $X \times[0,1)$. By sliding the $[0,1)-$ factor of the homotopy upward as the above, we have $g \gamma \simeq *$ in $X \times[0,1) \backslash L$. By the lifting property of $g$ [La], it follows that $\gamma \simeq *$ in $X \backslash f^{-1}(C)$. Thus we conclude that $f$ induces an isomorphism of homotopy groups of ends of $\operatorname{dim} \leq n$.

Proof of Theorem III. Let $f: M \rightarrow N$ be an $n$-homotopy equivalence between $\mu_{\infty}^{n+1}$-manifolds. Then by Lemma 3.1 there is a proper map $h: M \rightarrow N$ such that $f \simeq^{n} h$; consequently, $h$ is a proper $n$-homotopy equivalence. By Lemma 3.2 and Theorem 1.4, $h$ is properly $n$-homotopic to a homeomorphism. Thus $f$ is $n$-homotopic to a homeomorphism. 
Proof of Theorem IV. Let $f: M \rightarrow N$ be a map from a $\mu_{\infty}^{n+1}$-manifold to a $\mu^{n+1}$-manifold. By replacing $N$ with $\operatorname{Ker}(N)$, we may assume that $N$ is also a $\mu_{\infty}^{n+1}$-manifold. By the triangulation theorem for $\mu^{n+1}$-manifold [Dr], we can fix proper $(n+1)$-invertible $U V^{n}$-surjections $g: M \rightarrow K$ and $h: N \rightarrow L$, where $K$ and $L$ are locally finite polyhedra of dimension at most $n+1$. Then by the $(n+1)$-invertibility, $g$ has a section $p: K \rightarrow M$ (i.e., $g p=\operatorname{id}_{K}$ ). Since $N$ is a $\mu_{\infty}^{n+1}$-manifold, by Lemma 3.1, $f$ is $n$-homotopic to a proper map $f^{\prime}: M \rightarrow N$. Then $\varphi=h f^{\prime} p: K \rightarrow L$ is a proper map. Let $M(\varphi)$ be the mapping cylinder of $\varphi$, that is, a space obtained from the disjoint union $K \times[0,1] \oplus L$ by identifying $(x, 1)$ with $\varphi(x), x \in K$. Define $c_{\varphi}: M(\varphi) \rightarrow L$ by $c_{\varphi}(x, t)=\varphi(x)$, $x \in K$. Let $f_{n}: \mu^{n+1} \rightarrow Q$ be a proper $(n+1)$-invertible $U V^{n}$-surjection satisfying the condition $(*)$ in Theorem 1.2. Embed $M(\varphi)$ into $Q$, whence $f_{n}^{-1}(M(\varphi))$ is a $\mu^{n+1}$-manifold. We denote the restriction of $f_{n}$ to $f_{n}^{-1}(M(\varphi))$ by $f_{n} \mid$. Observe that $f_{n}^{-1}(K \times\{0\}) \cong M$ and $f_{n}^{-1}(L) \cong N$ by Proposition 1.3. We identify $f_{n}^{-1}(K \times\{0\}), f_{n}^{-1}(L)$ with $M, N$ respectively. Abusing notation, by $g: M \rightarrow K \times\{0\}, h: N \rightarrow L$ we denote the restrictions of $f_{n}$ to $M, N$ respectively. Using the $(n+1)$-invertibility of $h$, we can fix a section $q: L \rightarrow N$ of $h$. Note that since $c_{\varphi} f_{n} \mid: f_{n}^{-1}(M(\varphi)) \rightarrow L$ and $h: N \rightarrow L$ are proper $U V^{n}$-surjections, $f_{n}^{-1}(M(\varphi)) \cong N$ by Proposition 1.3. Observe that the map $q c_{\varphi} f_{n} \mid$ is an $n$-homotopy equivalence between $\mu_{\infty}^{n+1}$-manifolds $f_{n}^{-1}(M(\varphi))$ and $N$. Then by Theorem III, there is a homeomorphism $s$ : $f_{n}^{-1}(M(\varphi)) \rightarrow N$ such that $s \simeq^{n} q c_{\varphi} f_{n} \mid$. Note that $M^{\prime}=f_{n}^{-1}(K \times[0,1))$ is open in $f_{n}^{-1}(M(\varphi))$ and is a $\mu_{\infty}^{n+1}$-manifold by Theorem 2.1. Since the inclusion $i: M=f_{n}^{-1}(K \times\{0\}) \hookrightarrow M^{\prime}$ is an $n$-homotopy equivalence, by Theorem III, we can choose a homeomorphism $r: M \rightarrow M^{\prime}$ with $r \simeq^{n} i$. Then the map $s r: M \rightarrow N$ is an open embedding which is $n$-homotopic to $q c_{\varphi}\left(f_{n} \mid\right) i=q \varphi g=q h f^{\prime} p g$. Since $p g \simeq_{p}^{n} \mathrm{id}_{M}$ and $q h \simeq_{p}^{n} \mathrm{id}_{N}$, we have $q h f^{\prime} p g \simeq_{p}^{n} f^{\prime} \simeq^{n} f$. The proof is finished.

\section{ACKNOWLEDGMENT}

The author would like to thank Professor K. Sakai for his valuable suggestions.

\section{REFERENCES}

[Be] M. Bestvina, Characterizing k-dimensional universal Menger compacta, Mem. Amer. Math. Soc., vol. 71, no. 380, Amer. Math. Soc., Providence, RI, 1988.

[C1] T. A. Chapman, On some applications of infinite-dimensional manifolds to the theory of shape, Fund. Math. 74 (1972), 181-193.

[C2] _ On the structure of Hilbert cube manifolds, Compositio Math. 24 (1972), 329-353.

[C3] _ Lectures on Hilbert cube manifolds, C.B.M.S. Regional Conf. Ser. in Math., vol. 28, Amer. Math. Soc., Providence, RI, 1976.

[CS] T. A. Chapman and L. C. Siebenmann, Finding a boundary for a Hilbert cube manifold, Acta Math. 137 (1976), 171-208.

[Ch1] A. Chigogidze, Compacta lying in the n-dimansional universal Menger compactum and having homeomorphic complements in it, Mat. Sb. 133 (1987), 481-496; English transl., Math. USSR-Sb. 61 (1988), 471-484.

[Ch2] _ The theory of n-shapes, Uspekhi Mat. Nauk 44 (1989), 117-140; English transl., Russian Math. Surveys 44 (1989), 145-174. 
[Ch3] _ Classification theorem for Menger manifolds, Proc. Amer. Math. Soc. 116 (1992), 825-832.

[Ch4] _ $U V^{n}$-equivalence and n-equivalence, Topology Appl. 45 (1992), 283-291.

[Dr] A. N. Dranishnikov, Universal Menger compacta and universal maps, Mat. Sb. 129 (1986), 121-139; English transl., Math. USSR-Sb. 57 (1987), 131-150.

[Hu] Sze-Tsen Hu, Theory of retracts, Wayne State Univ. Press, Detroit, MI, 1965.

[La] R. C. Lacher, Cell-like mappings and their generalizations, Bull. Amer. Math. Soc. 83 (1977), 495-552.

[Wo] R. Y. T. Wong, Non-compact Hilbert cube manifolds, unpublished manuscript.

Institute of Mathematics, University of Tsukuba, Tsukuba-City, Ibaraki, 305 Japan

E-mail address: iwamotoy@sakura.cc.tsukuba.ac.jp 